$$
\text { No. } 4109 \text { July } 31,1948
$$

micro-second periods have been discovered which are produced by radioactive decay into stable isotopes ${ }^{6}$.

A study of the behaviour of nuclei immediately following nuclear reactions and inelastic collisions may show evidence of short-lived isomers. In order to exartine such effects, it is proposed, using a method similar to that here described, to irradiate various materials with neutrons produced by a pulsed ion beam in a $(D+D)$ generator.

Grateful acknowledgments are due to Dr. E. G. Bowen, chief of the Radiophysics Laboratory, for making available facilities for the experiments described, and to Dr. R. E. B. Makinson for suggesting this work. Financial assistance has been provided by a Commonwealth Science Research Grant.

School of Physics,

University of Sydney. March 30.

'Bowen, E. G., Pulley, O. O., and Gooden, J. S., Nature, 157, 840 (1946).

'Libby, W. F., and Lee, D. D., Phys. Rev., 55, 245 (1939).

${ }^{3}$ Waldman, B., and Collins, G. B., Phys. Rev., 57, 338 (L) (1940).

- Wiedenbeck, M. L., Phys. Rev., 67, 92 (1945).

- Waldman, B., Collins, G. B., Stubblefield, E. M., and Goldhaber, M., Phys. Rev., 55, 1129 (A) (1939).

- See, for example, de Benedetti, S., and McGowan, F. K., Phys. Rev., 70, 569 (1946); 71,380 (1947).

\section{A Gravitational Field with a Curious Geometrical Property}

IT is well known ${ }^{1}$ that there are fourteen independent absolute scalar differential invariants of the second order associated with the gravitational metric,

$$
d s^{2}=g_{\mu \nu} d x^{\mu} d x^{y} .
$$

It can be argued that the vanishing of all the invariants need not imply the vanishing of all the twenty independent components of the curvature tensor $R_{h i j k}$. On the other hand, it may be pointed out that, when the invariants vanish, there are fourteen differential equations to be satisfied by the ten $g_{\mu \nu}$ components; and it is not at all obvious that a gravitational field with a Riemannian metric exists for which the fourteen invariants vanish. We report here the existence of such a gravitational field described by the metric

$$
\begin{gathered}
d S^{2}=-A\left(d x^{2}+d y^{2}+d z^{2}-d t^{2}\right) \\
A=A(\xi), \xi=x-t,
\end{gathered}
$$

for which the surviving components of the energymomentum tensor satisfy the relations,

$$
T_{2}^{1}=T_{1}^{4}=-T_{4}^{1}=-T_{4}^{4},
$$

each being equal to

$$
\frac{1}{8 \pi}\left[\frac{1}{A^{2}} \frac{d^{2} A}{d \xi^{2}}-\frac{3}{2} \frac{1}{A^{3}}\left(\frac{d A}{d \xi}\right)^{2}\right] .
$$

The above metric with the conditions (3) may be compared to the gravitational field corresponding to a directed flow of radiation as given by Tolman ${ }^{2}$. For (2), the conformal curvature tensor vanishes and $T_{\mu}{ }^{\nu}$ has the structure of the electromagnetic energymomentum tensor; these circumstances together being responsible for the vanishing of the complete set of invariants.

\section{V. NARLIKaR}

K. R. KARMARKAR

Benares Hindu University. April 12.

'Thomas, T. Y., "Differential Invariants of Generalized Spaces", 183

"Tolman, $\dot{\boldsymbol{R}}$. C., "Relativity, Thermodynamics and Cosmology", 273

\section{Interpretation of Data from Electrical Resistivity Geophysical Surveys}

Mr. J. M. Hougr has described in Nature of May 22 a graphical method of analysing results from the four-electrode method of determining electrical resistivity, when applied to a horizontally stratified earth. He cites particularly the case of a two-layer earth. In a paper published in the Proceedings of the Physical Society, 47, 589 (1935), we described this graphical method and gave the universal curves for the case when the four electrodes are equally spaced. The analysis for the distribution of an altermating current with parallel flow in a two-layer earth was also given. Comparisons were made between results obtained with alternating currents and those obtained with the four-electrode method.

The graphical method just mentioned was used to some extent in the preparation of the electrical resistivity maps of Great Britain and southerm Scotland issued by the Electrical Research Association in 1935.

\section{S. WHITEHEAD}

British Electrical and Allied Industries

Research Association, Greenford, Middlesex.

General Post Office.

\section{W. G. RADLEY}

BEFORE I commenced an investigation of the interpretation of data from electrical resistivity surveys, unfortunately I failed to find any reference to the paper by Drs. Whitehead and Radley.

There are two points in the treatment of this method by Drs. Whitehead and Radley on which I should like to comment. They suggest that this method is not as accurate as that due to Tagg. For the ideal case of two perfectly homogeneous layers separated by a plane boundary parallel to a perfectly plane surface of the earth, this is probably true. In actual surveys, these conditions are never completely fulfilled, and deviations caused by irregularities in the boundary planes and inhomogeneities in the layers make any two-layer curve into only an approximation to the actual curve: under these conditions, I suggest that either method would be equally applicable.

In their paper, Drs. Whitehead and Radley only apply the method to a two-layer problem. I believe that the method is especially useful in the case of certain types of multi-layer earth. In my com. munication, I did not discuss this at length as the method is essentially the same as that proposed by Tagg, but, in my opinion, very much more convenient than that of Tagg. I have used the method on some results obtained by Prof. L. S. Palmer in Holderness for deposits which have three main layers, topsoil, low-resistance boulder clay (this layer is not usually homogeneous but contains thin strata of sand, etc.), and high-resistance chalk. In the eight cases examined, four showed a curve of three-layer type, and for these the specific resistance of the boulder clay ranged between 130 and $80 \mathrm{ohm} \mathrm{ft.,} \mathrm{and} \mathrm{that} \mathrm{of}$ the chalk between 240 and $570 \mathrm{ohm} \mathrm{ft}$. The topsoil varied from $2 \frac{1}{2} \mathrm{ft}$. and $6 \mathrm{ft}$. in thickness, and in two cases where bore-hole data were available the chalk was within 10 per cent of that for the bore-hole.

University College, J. M. Hougr Hull. 